\title{
Arabidopsis CNGC Family Members Contribute to Heavy Metal Ion Uptake in Plants
}

\author{
Ju Yeon Moon ${ }^{1}$, Célestine Belloeil ${ }^{1,2}$, Madeline Louise Ianna ${ }^{1,3}$ and Ryoung Shin ${ }^{1, *(1)}$ \\ 1 RIKEN Center for Sustainable Resource Science, 1-7-22 Suehirocho, Tsurumi-ku, Yokohama, \\ Kanagawa 230-0045, Japan; juyeon.moon@riken.jp (J.Y.M.); celestine.belloeil@gmail.com (C.B.); \\ mianna@myune.edu.au (M.L.I.) \\ 2 Université Paris Diderot, 5 rue Thomas Mann, 75013 Paris, France \\ 3 School of Science and Technology, UNE, Armidale, New South Wales 2351, Australia \\ * Correspondence: ryoung.shin@riken.jp; Tel.: +81-045-503-9577
}

Received: 30 November 2018; Accepted: 16 January 2019; Published: 18 January 2019

\begin{abstract}
Heavy metal ions, including toxic concentrations of essential ions, negatively affect diverse metabolic and cellular processes. Heavy metal ions are known to enter cells in a non-selective manner; however, few studies have examined the regulation of heavy metal ion transport. Plant cyclic nucleotide-gated channels (CNGCs), a type of $\mathrm{Ca}^{2+}$-permeable-channel, have been suggested to be involved in the uptake of both essential and toxic cations. To determine the candidates responsible for heavy metal ion transport, a series of Arabidopsis CNGC mutants were examined for their response to $\mathrm{Pb}^{2+}$ and $\mathrm{Cd}^{2+}$ ions. The primary focus was on root growth and the analysis of the concentration of heavy metals in plants. Results, based on the analysis of primary root length, indicated that AtCNGC1, AtCNGC10, AtCNGC13 and AtCNGC19 play roles in $\mathrm{Pb}^{2+}$ toxicity, while AtCNGC11, AtCNGC13, AtCNGC16 and AtCNGC20 function in $\mathrm{Cd}^{2+}$ toxicity in Arabidopsis. Ion content analysis verified that the mutations of AtCNGC1 and AtCNGC13 resulted in reduced $\mathrm{Pb}^{2+}$ accumulation, while the mutations of AtCNGC11, AtCNGC15 and AtCNGC19 resulted in less $\mathrm{Pb}^{2+}$ and $\mathrm{Cd}^{2+}$ accumulation in plants. These findings provide functional evidence which support the roles of these AtCNGCs in the uptake and transport of $\mathrm{Pb}^{2+}$ or $\mathrm{Cd}^{2+}$ ion in plants.
\end{abstract}

Keywords: Arabidopsis; cadmium; cyclic nucleotide-gated channel (CNGC); ion uptake; lead

\section{Introduction}

Toxic heavy metals (e.g., cadmium $(\mathrm{Cd})$, lead $(\mathrm{Pb})$, strontium $(\mathrm{Sr})$, or mercury $(\mathrm{Hg})$ ), as byproducts of rapidly growing industries, have been released into and accumulated in the soils at many sites, and have exerted adverse impacts on ecosystems [1-3]. Their detrimental effects have been attributed to their competition with other essential cations for binding to enzymes, inhibition of enzyme activity, or inducing the overproduction of reactive oxygen species (ROS), which leads to oxidative stress and potential cell death [3-5]. A number of other heavy metals (including iron $(\mathrm{Fe})$, copper $(\mathrm{Cu})$, zinc $(\mathrm{Zn})$, and nickel (Ni)), however, function as essential micronutrients in a variety of metabolic and cellular processes, such as primary/secondary metabolism, gene regulation, signal transduction, and hormone perception [6]. Essential metal ions, such as $\mathrm{Zn}^{2+}$ and $\mathrm{Fe}^{2+}$, are redox-active based and are involved in oxidation/reduction processes. An overabundance of these metals in cells, however, induces ROS formation. An excess of essential metals, as well as non-essential metals (e.g., $\mathrm{Cd}$ or $\mathrm{Pb}$ ), has an adverse effect on animal and plant cells. Therefore, organisms must maintain a fine-tuned homeostasis of the levels of heavy metals within a cell or have a mechanism that regulates their transport into/out of cells. However, the mechanism by which metal ions enter into plant and animal cells is relatively obscure. Non-essential toxic heavy metals can be non-selectively taken up by organisms largely because of 
their irrelevance to any known cellular function. Interestingly, some heavy metals, such as cesium (Cs) and $\mathrm{Sr}$ have physicochemical properties that are similar to the essential minerals potassium (K) and calcium (Ca) [7-11]. It has been shown that the radionuclide Cs likely enters plant cells through the K transport system [11].

Cyclic nucleotide-gated ion channels (CNGCs) have been suggested as potential candidates of ligand-gated/voltage-independent/cation transporters that play a role in the plant response to biotic/abiotic stresses, growth and development, and ion homeostasis [12-15]. CNGCs are activated (gated) by direct binding of cyclic nucleotides (CNs), such as cAMP (cyclic adenosine monophosphate) and cGMP (cyclic guanosine monophosphate), as well as by diverse molecules and ions, like $\mathrm{Ca}^{2+}$, $\mathrm{K}^{+}$, and $\mathrm{Na}^{+}$, and can be inactivated by binding $\mathrm{Ca}^{2+}$-activated calmodulin $(\mathrm{CaM})$ in a feedback mechanism [16-18]. In plants, CNGCs and Shaker-type $\mathrm{K}^{+}$-selective ion channels (voltage-dependent) have been postulated to be major targets of these ligands due to sharing some sequence homology and similarity in secondary structure. CN binding domains (CNBDs) are mainly present in the C-termini of plant CNGCs and Shaker-type $\mathrm{K}^{+}$channels coinciding with a CaM binding domain $[19,20]$. Despite their high similarity to Shaker-type voltage-gated channels, the heterologous expression of plant CNGCs in various yeast mutants deficient in ion uptake or efflux have demonstrated that CNGCs have a lower ion selectivity than Shaker-type channels and lower permeability to monovalent and divalent cations (such as $\mathrm{K}^{+}, \mathrm{Na}^{+}$, and $\mathrm{Ca}^{2+}$ ) across the plasma membrane [9,21-24]. After the first identification of a plant CNGC, barley HvCBT1 (Hordeum vulgare CaM-binding transporter), that binds to $\mathrm{CaM}$ in the C-terminus in a $\mathrm{Ca}^{2+}$-dependent manner [23], AtCNGC1 and AtCNGC2 were identified in Arabidopsis, and NtCBP4 was identified in tobacco (Nicotiana benthamiana). All were predicted to have a CaM-binding site at the CNBD in these CNGCs [25-28]. The Arabidopsis genome contains 20 CNGC family members that exhibit variable levels of expression in different tissues [15]. The CNGC family is divided into five subfamily groups (I, II, III, IV-A and IV-B) based on their sequence similarity. Group I, II, and III are closely related, whereas groups IV-A and IV-B are distantly related to each other and to the other groups [29].

Heterologous expression systems and mutant plants have been used to characterize the functional roles of CNGC family members in ion transport across plant cells. Complementation analysis using $\mathrm{K}^{+}$- and $\mathrm{Ca}^{2+}$-uptake-deficient yeast mutants demonstrated that AtCNGC1 and AtCNGC2 partially recover $\mathrm{K}^{+}$uptake [27], and that the AtCNGC11 and AtCNGC12 channels are permeable to $\mathrm{Ca}^{2+}[30]$. Similarly, Leng et al. described AtCNGC2 as a CN-mediated cation channel that is permeable to $\mathrm{Ca}^{2+}$ and $\mathrm{K}^{+}$, but not $\mathrm{Na}^{+}[21,22]$. When AtCNGC2 was introduced into Xenopus laevis oocytes lacking a low-affinity $\mathrm{K}^{+}$uptake system or human embryonic kidney cells (HEK293), they displayed $\mathrm{CN}$-dependent, inward-rectifying $\mathrm{K}^{+}$currents and increased permeability to $\mathrm{Ca}^{2+}$ only in the presence of the CNs [21,22]. Electrophysiological studies of AtCNGC1 and AtCNGC4 suggested that they function in the uptake of monocations (such as $\mathrm{K}^{+}$and $\mathrm{Na}^{+}$) [21,31]. In a reverse genetic study, a atcngc2 knockout mutant exhibited hypersensitivity to $\mathrm{Ca}^{2+}$ but not to $\mathrm{K}^{+}$or $\mathrm{Na}^{+}$[32]. In addition, the atcngc1 mutant also had a lower level of $\mathrm{Ca}^{2+}$ in shoots than wild-type plants [33]. Atcngc3 mutant seedlings exhibited less sensitivity in their growth response to toxic levels of $\mathrm{K}^{+}$and $\mathrm{Na}^{+}$and lower cation levels in their tissues, relative to wild-type plants, indicating that AtCNGC3 plays a role in the non-selective uptake of monovalent cations [34]. AtCNGC10 expression rescued the $\mathrm{K}^{+}$uptake channel mutant, akt1 (Arabidopsis Shaker-type $\mathrm{K}^{+}$channel, AKT1), as well as similar Escherichia coli and yeast mutants, suggesting that AtCNGC10 likely mediates $\mathrm{K}^{+}$influx into cells. AtCNGC10 overexpression results in 1.7-fold better growth of the $a k t 1$ mutant under $\mathrm{K}^{+}$-limited conditions [35]. Although sequence similarity among the members of AtCNGC family ranges between $55 \%$ and $83 \%$ [29], they have exhibited discernible levels of specificity in the transport of diverse cations, and thus, the differences confer independent phenotypes with AtCNGC mutants.

Due to their potential non-selectivity in the uptake of different cations, plant CNGCs have been predicted to also be involved in the uptake of micronutrient ions into cells, as well as toxic ions [34,36,37]. Nuclear magnetic resonance spectroscopy and chemical studies have been used to 
investigate the potential binding of $\mathrm{Mg}^{2+}, \mathrm{Sr}^{2+}$, or $\mathrm{Pb}^{2+}$ to $\mathrm{Ca}^{2+}$-binding sites of $\mathrm{CaM}$ in a distinct manner indicating an inhibitory effect on the interactions between $\mathrm{CaM}$ and functional targets, e.g., $\mathrm{Ca}^{2+}$ ion $[38,39]$. Interestingly, $\mathrm{Pb}^{2+}$ ion has been reported to enter into animal cells (bovine adrenal medullary cells or chromaffin cells) through $\mathrm{Ca}^{2+}$ channels [40,41]. Sunkar et al. reported that the expression of a truncated NtCBP4 in transgenic tobacco and a mutation in AtCNGC1 contribute to increased tolerance to $\mathrm{Pb}^{2+}$ [37]. In contrast, $\mathrm{NtCBP} 4$ overexpression causes hypersensitivity to $\mathrm{Pb}^{2+}$ ion but tolerance to $\mathrm{Ni}^{2+}$ [26]. AtCNGC2 provides conductivity to other monovalent cations $\left(\mathrm{Li}^{+}, \mathrm{Cs}^{+}\right.$, and $\mathrm{Rb}^{+}$), as high as to $\mathrm{K}^{+}$ion, but much lower conductivity to $\mathrm{Na}^{2+}$ ion [21]. AtCNGC10 was also reported to regulate the transport of $\mathrm{Mg}^{2+}$ as well as $\mathrm{Ca}^{2+}$ ion in the shoots and roots of Arabidopsis plants. Based on studies utilizing heterologous expression and knockout systems, a consensus is developing that plant CNGCs can modulate the flux of a wide range of ions into plant cells. We have been interested in the relationship between members of the AtCNGC family and the uptake of diverse cations. In the current study, we examined the effect of two toxic heavy metal ions, $\mathrm{Cd}^{2+}$ and $\mathrm{Pb}^{2+}$, on different Arabidopsis atcngc mutant plants. Root growth and ion levels were analyzed to compare differences in the responses among the mutant lines and wild-type plants to the application of heavy metals.

\section{Results}

\subsection{Primary Root Growth Analysis in $\mathrm{Pb}^{2+}$ Stressed atcngc Mutant Plants}

The growth of primary roots in Arabidopsis mutants of all AtCNGC members, except AtCNGC9 and AtCNGC18, were measured to determine their response to heavy metal ions, relative to the response of Col-0 wild-type plants. One or more Arabidopsis mutation alleles for the eighteen distinct AtCNGC genes were used in this study (Figure 1). PCR assay and sequencing analysis were performed to clarify the genotypes and single nucleotide subsitution mutations, and some of PCR results present in Figure S1. The seeds of the mutant and wild-type plants used in this study were germinated and grown for eight days with or without $150 \mu \mathrm{M} \mathrm{Pb}\left(\mathrm{NO}_{3}\right)_{2}$. Then, the lengths of the primary root of plants were measured and compared between seedling plants grown on media with $\mathrm{Pb}^{2+}$ versus seedlings grown on media without $\mathrm{Pb}^{2+}$ ion. The results indicated that all of the eight-day-old seedlings grown on media with $\mathrm{Pb}^{2+}$ exhibited decreased primary root growth relative to roots of seedlings grown on media without $\mathrm{Pb}^{2+}$, although the original lengths of primary roots differed between different Arabidopsis mutants without the heavy metal (Figure 2). To more clearly define the level of root growth inhibition due to $\mathrm{Pb}^{2+}$, a finer measure of primary root length was made using ImageJ software, and statistical analyses of the effect of $\mathrm{Pb}^{2+}$ and differences between the mutant lines were conducted (Figure 2). Results indicated that the mutant lines of AtCNGC1 (CS874223, atcngc1-1), AtCNGC10 (CS859870, atcngc10-1), and AtCNGC13 (Salk_057742, atcngc13-1; Salk_013536, atcngc13-2) exhibited less growth inhibition in response to $\mathrm{Pb}^{2+}$, relative to the level of inhibition in the wild-type plants. In addition, one of the AtCNGC19 mutant alleles, atcngc19-1, presented a shorter primary root compared to that of the wild-type or the other AtCNGC19 mutant, atcngc19-2, in the absence of $\mathrm{Pb}^{2+}$, while the three different types of plant were grown with comparable growth size in the presence of $\mathrm{Pb}^{2+}$ (Figure 2). This relatively smaller growth reduction (root growth in $+\mathrm{Pb}^{2+} /-\mathrm{Pb}^{2+}$ ) of atcngc19-1 in the presence of $\mathrm{Pb}^{2+}$ indicates that the mutation in AtCNGC19 (CS860128, atcngc19-1) caused less negative effect on $\mathrm{Pb}^{2+}$-stressed Arabidopsis plants. The other AtCNGC19 mutant allele (CS860131 atcngc19-2) showed a similar root length to the wild-type plants regardless of $\mathrm{Pb}^{2+}$ treatment. Mutants of the other AtCNGC members exhibited similar levels of growth inhibition to that of the wild-type (Figure S2). These data indicate that plants lacking AtCNGC1, AtCNGC10, AtCNGC13, or AtCNGC19 exhibited increased tolerance to $\mathrm{Pb}^{2+}$ toxicity, and that these AtCNGC members are involved in $\mathrm{Pb}^{2+}$ uptake into plants. In contrast, plants lacking AtCNGC15 (CS93704, atcngc15-1) or AtCNGC11 (Salk_026568, atcngc11-1) exhibited increased inhibition of primary root growth relative to the wild-type. These data indicate that in contrast to the four previously described AtCNGC members, AtCNGC11 and 
AtCNGC15 function positively in regulating plant tolerance to $\mathrm{Pb}^{2+}$ stress (Figure 2). However, the other AtCNGC11 mutant allele (Salk_085485, atcngc11-2) did not show significantly different root growth in response to $\mathrm{Pb}^{2+}$ compared to the wild-type. The Transfer-DNA (T-DNA) insertion in atcngc11-1 locates between the seventh exon and the eighth exon, whereas the insertion in atcngc11-2 locates in the $5^{\prime}$-UTR region. The different root growth responses to $\mathrm{Pb}^{2+}$ in atcngc11 mutants may be due to the different locations of T-DNA insertions, but further confirmation is required.

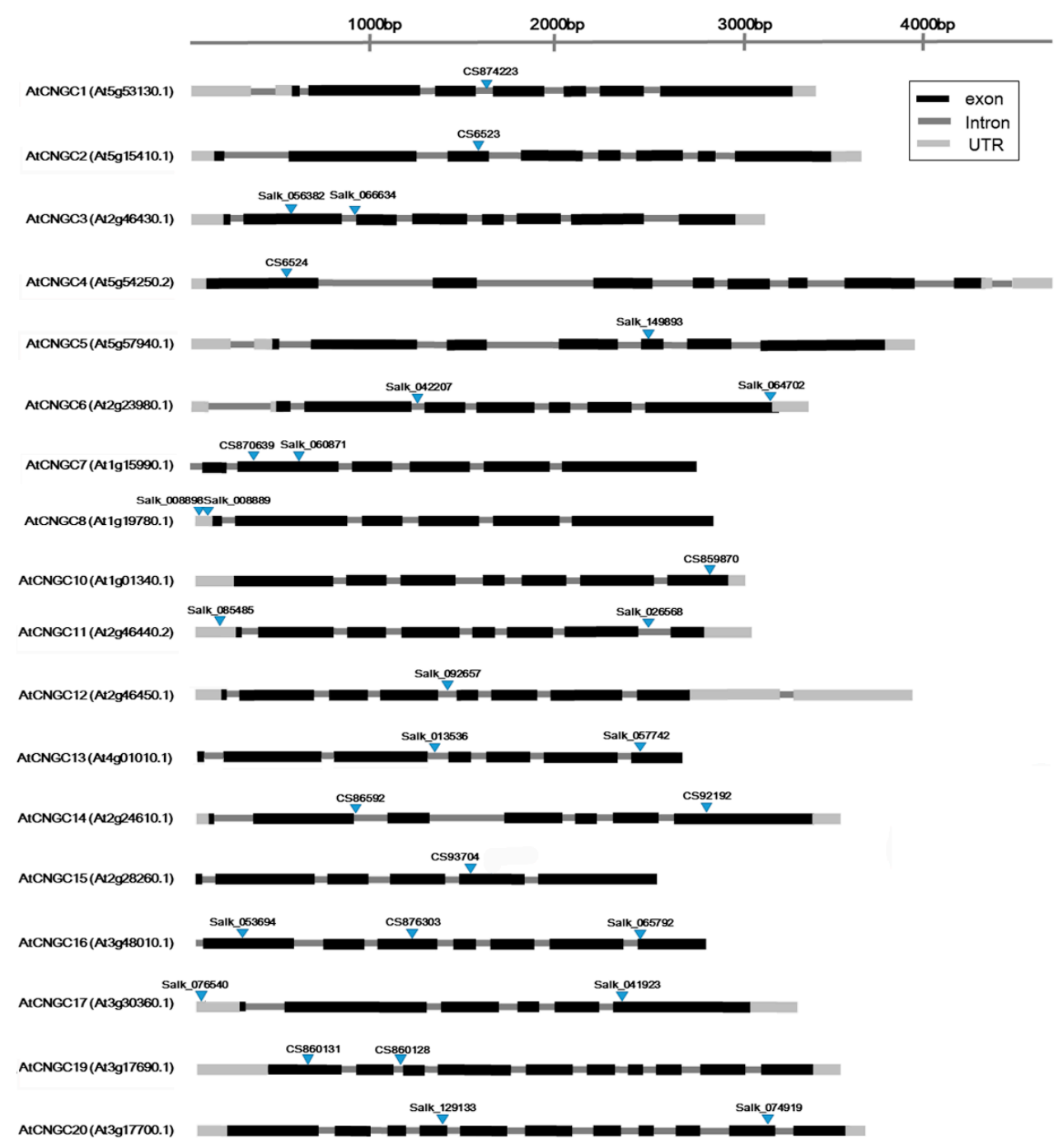

Figure 1. Schematic diagram illustrating the structure of the Arabidopsis CNGC family genes. The exon-intron structure in each of the genes is presented and the loci of the T-DNA insertions or single nucleotide substitutions (for AtCNGC2, AtCNGC4, AtCNGC14 and AtCNGC15) in the atcngC knockout mutants are marked with upside-down triangles. The Arabidopsis mutant seeds were obtained from the Arabidopsis Biological Resource Center (ABRC) (https://abrc.osu.edu/): Light grey: UTR, dark grey: intron, black: exon. 


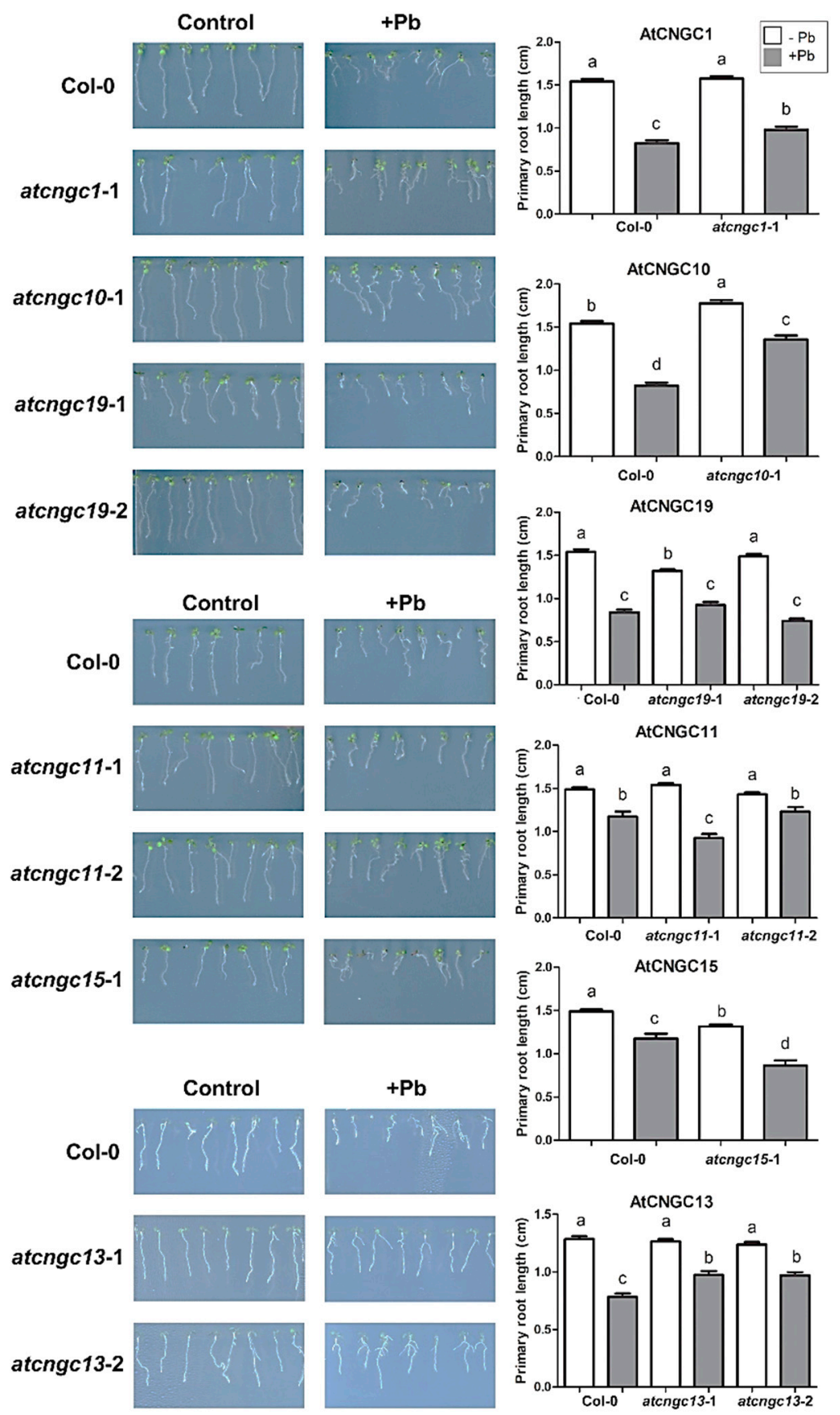

Figure 2. Growth of the Arabidopsis wild-type and atcngc mutants in response to $\mathrm{Pb}^{2+}$. Eight-day-old seedlings grown on agar media containing $150 \mu \mathrm{M} \mathrm{Pb}\left(\mathrm{NO}_{3}\right)_{2}$ were photographed and were used to measure primary root growth (length) using ImageJ software. The presented images (left side of figure) are representative of $\geq 80$ seedlings of each genotype (the mutant or the wild-type) used to measure the primary root length for each treatment (right side of figure). Quantitative data presented represents the mean \pm SEM. Data were subjected to a one-way ANOVA with a post-hoc Tukey's comparison. Different letters indicate significant differences $(p<0.05)$.

\subsection{Primary Root Growth Analysis in $\mathrm{Cd}^{2+}$ Stressed atcngc Mutant Plants}

Cadmium is also considered to be a very toxic heavy metal, with deleterious impacts on plant growth and the environment. Primary root length was also measured in eight-day-old wild-type and atcngc mutant lines grown on agar media with or without $55 \mu \mathrm{M} \mathrm{CdCl}_{2}$. The eight-day-old seedlings grown on agar media with $\mathrm{Cd}^{2+}$ appeared less healthy than seedlings grown without $\mathrm{Cd}^{2+}$ (Figure 3 ). 
Relative to the response of the wild-type plants, however, four different AtCNGC mutants were observed to have less growth retardation from the $\mathrm{Cd}^{2+}$ ion. Plants lacking AtCNGC11 (Salk_026568, atcngc11-1; Salk_085485, atcngc11-2), and AtCNGC16 (Salk_065792, atcngc16-1; Salk_053694, atcngc16-2) exhibited relatively less inhibition of primary root growth, while other AtCNGC mutants displayed similar levels of root growth inhibition as that of the wild-type (Figure 3 and Figure S3). Moreover, AtCNGC13 (Salk_057742, atcngc13-1) or AtCNGC20 (Salk_129133, atcngc20-1) have shorter primary roots relative to that of the wild-type in absence of $\mathrm{Cd}^{2+}$, however, the primary root lengths of AtCNGC mutants and the wild-type plants were comparable in presence of $\mathrm{Cd}^{2+}$, indicating that the less levels of growth inhibition in the atcngc13-1 and atcngc20-1 mutants by $\mathrm{Cd}^{2+}$ stress. The lower levels of primary root growth inhibition in the plants with the mutant alleles indicates the possibility that AtCNGC11, AtCNGC13, AtCNGC16, and AtCNGC20 function as positive components of $\mathrm{Cd}^{2+}$ uptake, similar to the result observed with $\mathrm{Pb}^{2+}$ (Figure 2). The mutant allele for AtCNGC13, atcngc13-1, displayed a lower level of primary root growth inhibition in response to both $\mathrm{Cd}^{2+}$ and $\mathrm{Pb}^{2+}$. These data suggest that AtCNGC13 functions negatively in the plant response to the two different heavy metal ion-contaminated conditions.

\subsection{Quantitative Assessment of $\mathrm{Pb}^{2+}$ Levels in atcngc Mutant Plants}

All of the Arabidopsis plantlets that were used to measure primary root length were collected and analyzed for heavy metal ion content to determine if the alteration in growth response to $\mathrm{Pb}^{2+}$ in the atcngc mutants was related to differential accumulation of heavy metals between the wild-type and mutant plants. The contents of the $\mathrm{Ca}^{2+}, \mathrm{K}^{+}, \mathrm{Pb}^{2+}$ and $\mathrm{Cd}^{2+}$ ions were determined using an ICP-MS system, and the final value was calculated based on dry weight. $\mathrm{K}^{+}$levels in the wild-type system, and the final value was calculated based on the dry weight. $\mathrm{K}^{+}$levels in the wild-type versus atcngc mutant plantlets were not significantly different among the wild-type and AtCNGC mutants, although the $\mathrm{Ca}^{2+}$ content in some atcngc mutant lines was slightly lower than that in the wild-type (Figure S4). Consistent with the phenotypic analysis presented in Figure 2, a lower concentration of $\mathrm{Pb}^{2+}$ ion was found in the atcngc1-1, atcngc13-1, and atcngc19-1 mutants, but not in the atcngc10-1 mutant, than in the wild-type plants (Figure 4); indicating that the lower level of primary root growth inhibition was, at least in part, the result of a lower accumulation of $\mathrm{Pb}^{2+}$ in the mutant plants. The atcngc13-1 and atcngc19-1 mutant plants, however, exhibited less and similar growth retardation, respectively, to the wild-type; while $\mathrm{Pb}^{2+}$ ion accumulation in the atcngc13-2 mutant and atcngc19-2 mutant, respectively, was either comparable or lower than what was found in the wild-type plants (Figures 2 and 4). Additionally, atcngc11-1 and atcngc15-1 mutants also had lower levels of $\mathrm{Pb}^{2+}$ than the wild-type plants, despite exhibiting a higher level of primary root growth inhibition than the wild-type plants when grown in the presence of $\mathrm{Pb}^{2+}$ (Figures 2 and 4). This indicated that AtCNGC11 or AtCNGC15 have possible roles in $\mathrm{Pb}^{2+}$ ion uptake, but the further inhibition of root growth in their mutants by $\mathrm{Pb}^{2+}$ treatment may not directly result from a lower $\mathrm{Pb}^{2+}$ content in the mutant plants. Collectively, the quantitative analysis of heavy metal content confirmed the relationship between the growth defect phenotype and reduced $\mathrm{Pb}^{2+}$ ion levels in Arabidopsis atcngc1, atcngc13, and atcngc19 seedlings. In addition, these data indicate that at least AtCNGC13 and AtCNGC19 as well as AtCNGC1 appear to be involved in $\mathrm{Pb}^{2+}$ ion uptake into plant cells. These results are consistent with a previous study on AtCNGC1-related $\mathrm{Pb}^{2+}$ ion uptake [37]. 


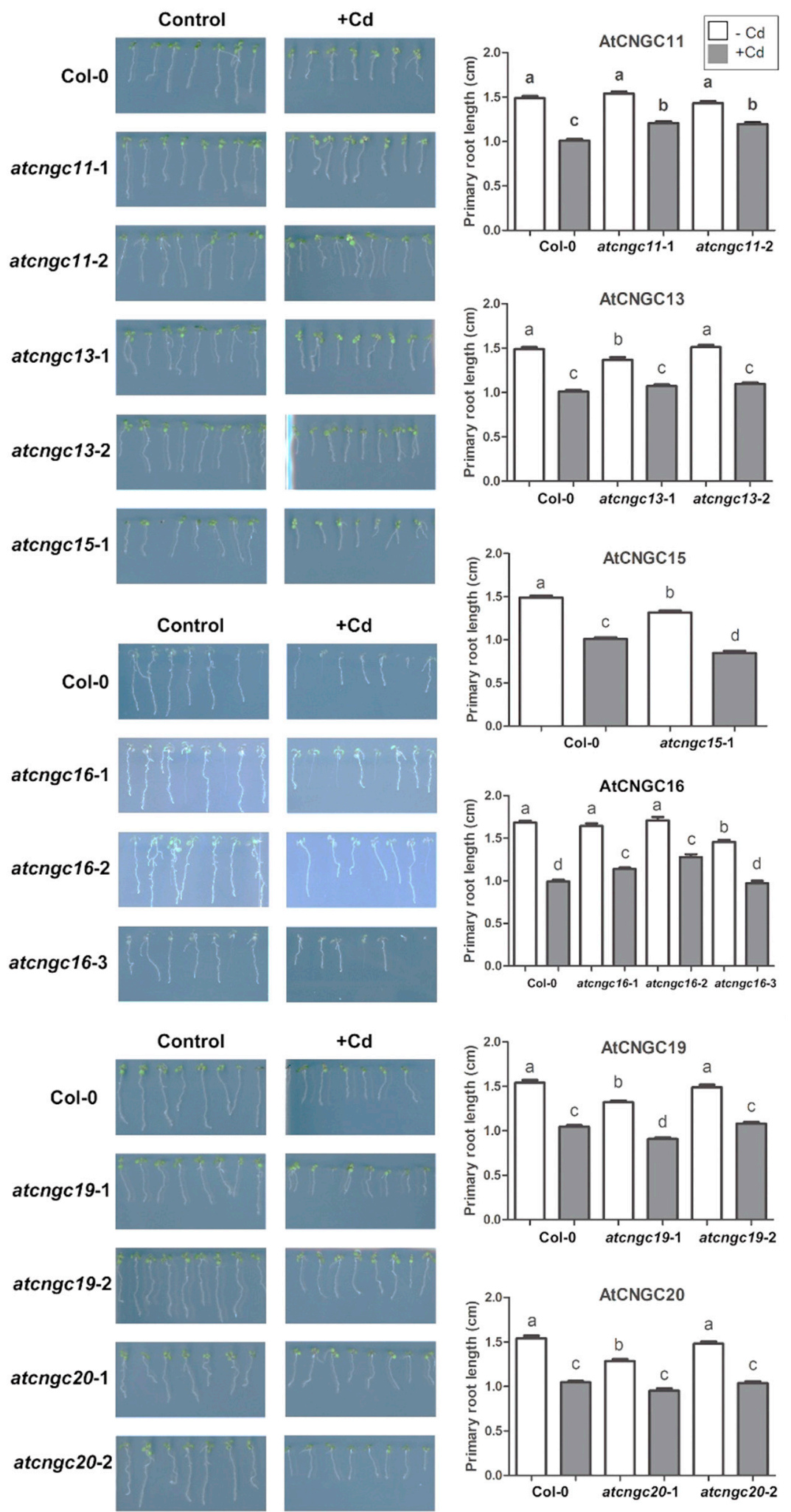

Figure 3. Growth of wild-type Arabidopsis and atcngc mutants in response to $\mathrm{Cd}^{2+}$. Eight-day-old seedlings grown on agar media supplemented with $55 \mu \mathrm{M} \mathrm{CdCl}_{2}$ were photographed and used to measure primary root growth (length) using ImageJ software. The presented images (left side of figure) are representatives of $\geq 80$ seedlings of each genotype (the mutant or the wild-type) used to measure each treatment (right side of figure). Quantitative data presented represent the mean \pm SEM. Data were subjected to a one-way ANOVA with a post-hoc Tukey's comparison. Different letters indicate significant differences $(p<0.05)$. 

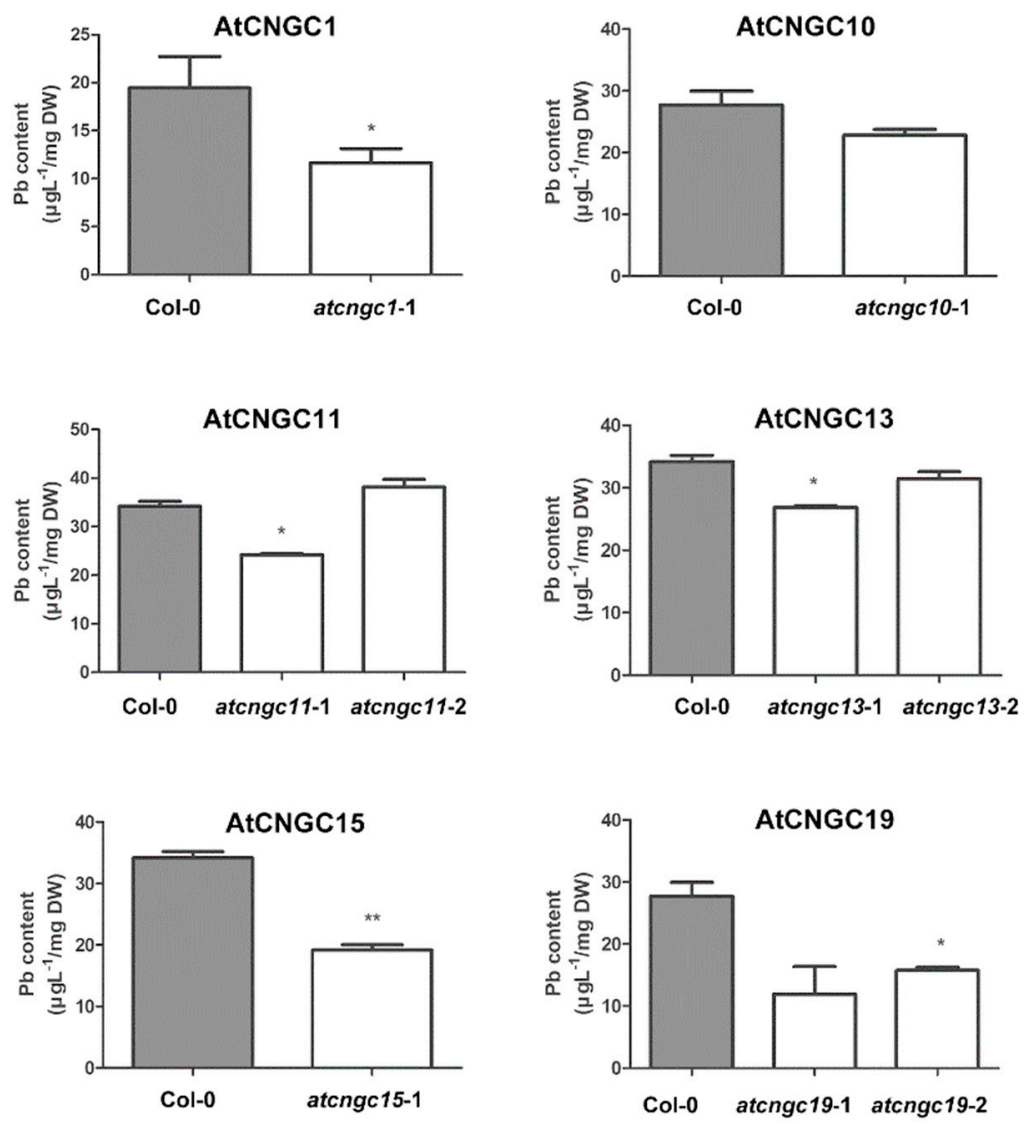

Figure 4. $\mathrm{Pb}^{2+}$ content in Arabidopsis plants grown on an agar medium with $\mathrm{Pb}^{2+}$. All of the seedlings used to measure primary root growth in three replicated experiments were combined and extracted in $60 \%(v / v) \mathrm{HNO}_{3}$. Elemental analysis was conducted utilizing an ICP-MS System. Data presented represent the mean $\pm \operatorname{SEM}(n=3)$. T-tests were conducted to compare the concentration of each element in $c n g c$ mutants vs. wild-type plants. ${ }^{*}$ and ${ }^{* *}$ symbols indicate significant differences at $p>0.05$ and $p>0.01$, respectively.

\subsection{Quantitative Assessment of $\mathrm{Cd}^{2+}$ Levels in atcngc Mutant Plants}

$\mathrm{Cd}^{2+}$ content was also assessed in the eight-day-old mutant and wild-type plantlets grown in the presence of $\mathrm{Cd}^{2+}$ ion that were used to measure primary root length. Among the atcngc mutants that exhibited a lower inhibition of primary root growth, $\mathrm{Cd}^{2+}$ ion accumulation was lower only in the atcngc11-1, and atcngc11-2 mutants than it was in the wild-type plantlets; while the $\mathrm{Cd}^{2+}$ levels in the atcngc13-1 and atcngc20-1 mutants and the three atcngc16 mutants were not significantly different than the level found in the wild-type plants (Figures 3 and 5). Taken together, it is probable that AtCNGC11 has a role in $\mathrm{Cd}^{2+}$ ion entry into plants, and thus negatively affects plant tolerance to $\mathrm{Cd}^{2+}$ stress. Notably, atcngc15-1, as well as atcngc19-1 and atcngc19-2, mutants exhibited less $\mathrm{Cd}^{2+}$ ion accumulation in the plantlets, although no differences in the level of primary root growth inhibition relative to wild-type plants were observed. This may be due to their shorter primary root lengths than those of wild-type plants even without $\mathrm{Cd}^{2+}$ treatment. These data suggest that AtCNGC15 and AtCNGC19 may have some relationship to $\mathrm{Cd}^{2+}$ ion uptake, but also in the regulation of root development resulting from $\mathrm{Cd}^{2+}$ contamination. Collectively, the data indicate that AtCNGC11 and possibly AtCNGC15 and AtCNGC19 are potential components of $\mathrm{Cd}^{2+}$ ion uptake into plant cells. 

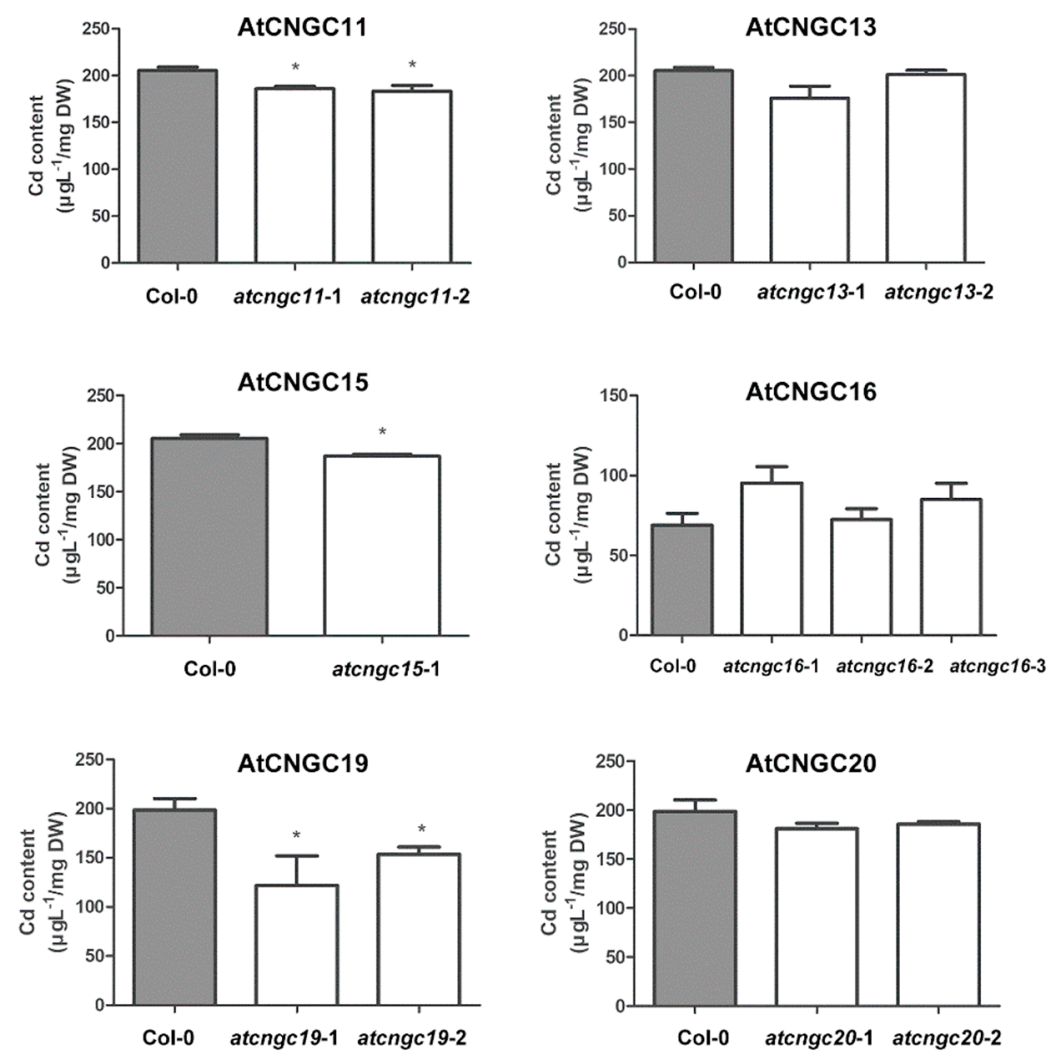

Figure 5. $\mathrm{Cd}^{2+}$ content in Arabidopsis plants grown on an agar medium with $\mathrm{Cd}^{2+}$. All of the seedlings used to measure primary root growth in three replicated experiments were combined and extracted in $60 \%(v / v) \mathrm{HNO}_{3}$. Elemental analysis was conducted utilizing an ICP-MS System. Data presented represent the mean $\pm \operatorname{SEM}(n=3)$. T-tests were conducted to compare the concentration of each element in $c n g c$ mutants vs. wild-type plants. ${ }^{*}$ and ${ }^{* *}$ symbols indicate significant differences at $p>0.05$ and $p>0.01$, respectively.

\section{Discussion}

The CNGC proteins are typically known to $\mathrm{Ca}^{2+}$-permeable channels involved in the uptake of diverse monovalent cations and several CNGC members also display the ability to mediate the transport of divalent cations $[21,22,30,31,34,36]$. Apart from a study on the positive participation of AtCNGC1 in $\mathrm{Pb}^{2+}$ ion uptake into plants, relatively little information exists on the role of Arabidopsis AtCNGC members in the uptake of toxic heavy metal ions. In the present study, the potential role of the various Arabidopsis CNGC family members in ion uptake was investigated, especially the heavy metal $\mathrm{Pb}^{2+}$ and $\mathrm{Cd}^{2+}$ ions by utilizing a series of atcngc mutants. The results indicated that the knockout of specific AtCNGC genes confers tolerance to $\mathrm{Pb}^{2+}$ or $\mathrm{Cd}^{2+}$ ion as measured by lower inhibition of primary root growth. Lower inhibition of primary root growth phenotypes with less accumulation of heavy metal ions was observed in several atcngc mutants. The results indicated that AtCNGC1, AtCNGC10, AtCNGC13, and AtCNGC19 have negative effects on $\mathrm{Pb}^{2+}$ stress, while AtCNGC11, AtCNGC13, AtCNGC16, and AtCNGC20 have negative effects on $\mathrm{Cd}^{2+}$ stress. Therefore, AtCNGC13 functions as a negative component in plant tolerance to both $\mathrm{Pb}^{2+}$ and $\mathrm{Cd}^{2}$. Conversely, AtCNGC11 and AtCNGC15 appear to be positively involved in plant tolerance to $\mathrm{Pb}^{2+}$ in Arabidopsis (Figures 2 and 3).

The analysis of heavy metal content indicated that AtCNGC11 and AtCNGC15 as well as AtCNGC1, AtCNGC13, and AtCNGC19 are potential factors in the uptake mechanism of $\mathrm{Pb}^{2+}$ ion into plant cells, while AtCNGC11, AtCNGC15, and AtCNGC19 are likely involved in $\mathrm{Cd}^{2+}$ uptake (Figures 4 and 5). Unlike AtCNGC1, AtCNGC13, or AtCNGC19, the mutation of either AtCNGC11 (atcngc11-1) or AtCNGC15 (atcngc15-1) confers less tolerance to $\mathrm{Pb}^{2+}$ stress than the wild-type (Figure 2). 
Differences in the obtained results with the various AtCNGC members may be partially due to the ability of AtCNGC11 or AtCNGC15 to indirectly activate the other AtCNGCs or other ion transport channels that compensate for the lack of the ions in plants. Thus, the activated channels cause a more severe phenotype by enhancing toxic ion uptake. This speculation, however, needs to be further investigated with additional molecular and biological studies. To the best of our knowledge, the present study is the first to identify several AtCNGC members as potential components in the plant response to the heavy metal ions $\mathrm{Pb}^{2+}$ and $\mathrm{Cd}^{2+}$ and, more specifically, the uptake of $\mathrm{Pb}^{2+}$ and $\mathrm{Cd}^{2+}$ ions. Importantly, while knockout of AtCNGC10 rendered Arabidopsis plants more tolerant to $\mathrm{Pb}^{2+}$, and knockout of $A t C N G C 13, A t C N G C 16$, or $A t C N G C 20$ resulted in greater tolerance to $\mathrm{Cd}^{2+}$ treatment, respectively, no significant difference in heavy metal contents was confirmed in the mutant plants relative to the wild-type plants. This discrepancy between the plant root growth and the heavy ions content in the four different AtCNGC mutants indicates that these specific AtCNGC members are not directly involved in $\mathrm{Cd}^{2+}$ or $\mathrm{Pb}^{2+}$ ion uptake into plant cells but rather, function in a negative manner in regard to plant tolerance to heavy metals.

In the present study, there was a discrepancy in the phenotypic and heavy metal ion content results obtained using two different mutation alleles of the same AtCNGC genes (e.g., atcngc13-1, atcngc13-2 on $\mathrm{Pb}^{2+}$ and $\mathrm{Cd}^{2+}$ stress). AtCNGC members are known to be activated through the binding of signal molecules, such as cyclic nucleotides, on CNBDs; however, the different responses to $\mathrm{Pb}^{2+}$ and/or $\mathrm{Cd}^{2+}$ in mutants of the same AtCNGCs (e.g., atcngc13-1 and atcngc13-2) are likely due to the different locations of the T-DNA insertion (Figure 1). However, the obvious developmental defects of the AtCNGC mutants have not been revealed except for the atcngc2 and atcngc4 mutants showing severe growth phenotype and infertility [42]. Despite some mutant lines exhibiting a similar level of inhibition of primary root growth in response to $\mathrm{Pb}^{2+}$ and $\mathrm{Cd}^{2+}$ as the wild-type plants, most of the AtCNGC mutants accumulated fewer heavy metals. This strongly suggests that AtCNGC members play a functional role in the uptake process for $\mathrm{Cd}^{2+}$ or $\mathrm{Pb}^{2+}$ ions.

Twenty Arabidopsis CNGC members share 55\% to 83\% sequence similarity, and are divided into five evolutionary groups based on the alignment of the predicted amino acid sequences. These subclasses are designated as groups I, II, III, IV-A, and IV-B [29]. In the present study, among the AtCNGC members proposed to function in $\mathrm{Pb}^{2+}$ ion uptake, AtCNGC1, AtCNGC11, and AtCNGC13 are in group I, while AtCNGC15 and AtCNGC19 belong to group III and IV-A, respectively (Figure 6). Interestingly, $\mathrm{NtCBP4}$, a tobacco CNGC, has also been reported to be a component of $\mathrm{Pb}^{2+}$ ion uptake in plants, and is closely related to AtCNGC1 based on the alignment of their protein sequences $[29,37]$. Since AtCNGC and the tobacco NtCBP4 have been suggested to share a common ancestor [29], AtCNGC11 and AtCNGC13 may have also been derived from the same ancestor as NtCBP4. As group I also includes AtCNGC11, which has also been implicated as a potential transporter of $\mathrm{Cd}^{2+}$, other AtCNGC members may have potential roles as components of $\mathrm{Cd}^{2+}$ transport. AtCNGC16 and AtCNGC20, which exhibit a negative relationship to the plant tolerance response to $\mathrm{Cd}^{2+}$, are members of group II and IV-A, respectively (Figure 6). Notably, none of the AtCNGCs in group II and IV-B have been identified as components of plant tolerance to heavy metals or heavy metal ion uptake. This suggests that the AtCNGCs in these distinct groups have different characteristics in regard to their roles in heavy metal uptake. 


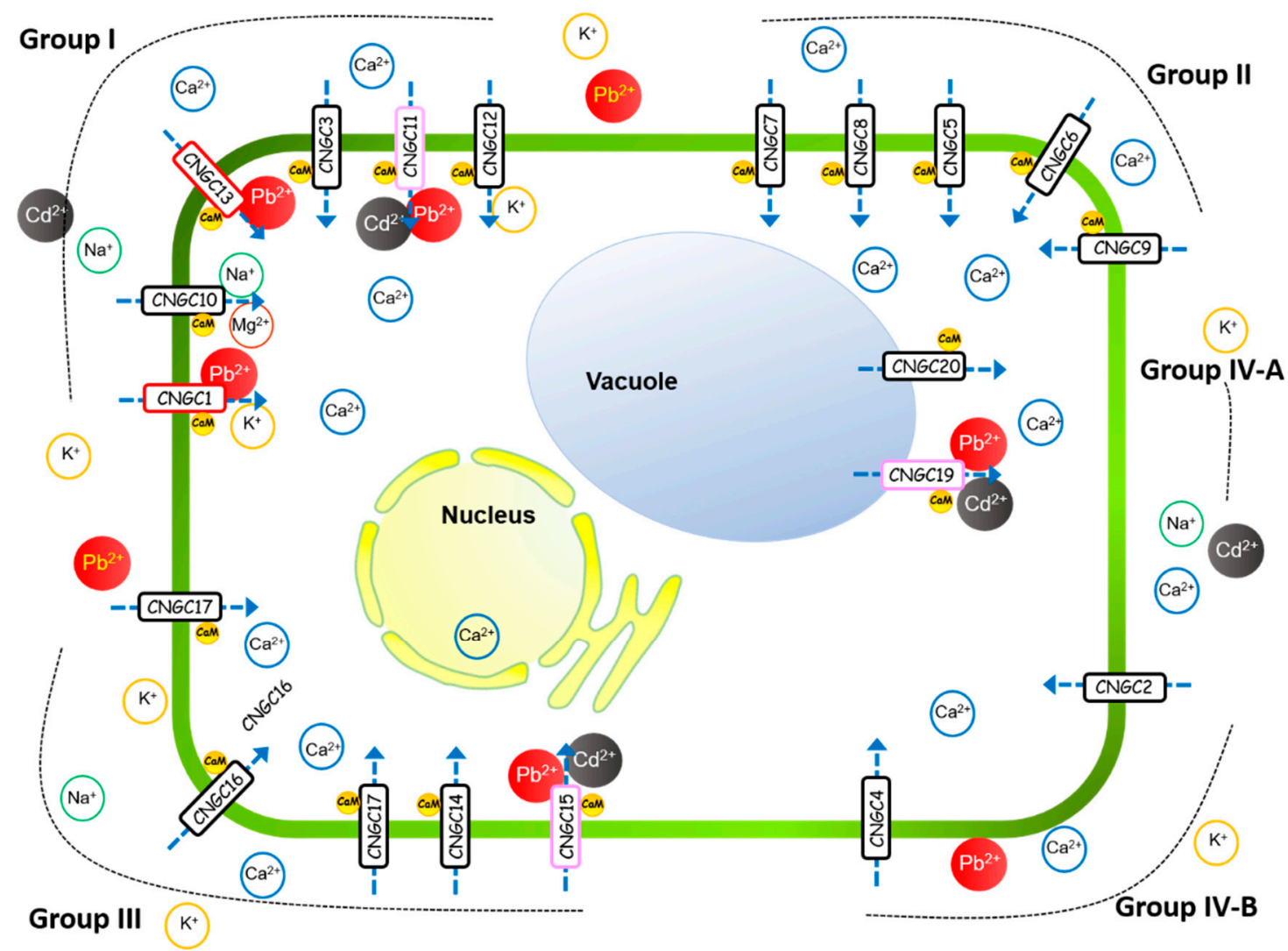

Figure 6. Model of the involvement of AtCNGC family members in ion transport in plants. AtCNGC members are divided in five groups according to the alignment of their predicted amino acid sequences, and locate to the plasma membrane (group I, II, III, IV-B) or vacuolar membranes (IV-A) (drawn as adjacent shapes with arrows) [12,29]. Ions are transported with the help of $\mathrm{Ca}^{2+}$-permeable AtCNGC family members dependent on the different ions. AtCNGCs in red rectangle: for $\mathrm{Pb}^{2+}$ entry, AtCNGCs in pink rectangle: for $\mathrm{Pb}^{2+}$ or $\mathrm{Cd}^{2+}$ entry.

Arabidopsis CNGCs have been generally hypothesized to localize to the plasma membrane, although AtCNGC19 and AtCNGC20 have been previously reported to localize to the tonoplast; suggesting that they function as passive transporters of cations between the vacuole and cytosol $[12,43]$. Toxic ions are preferentially sequestered into the vacuole to counteract their toxicity in the cytosol [44]. In previous studies, genes encoding AtCNGC19 and AtCNGC20 were upregulated in response to salinity stress and it was suggested that AtCNGC19 and AtCNGC20 function to mediate the plant response to salt stress by mediating $\mathrm{Ca}^{2+}$ signaling $[43,45,46]$. Despite the data in the present study indicating a negative role for AtCNGC20 in plant tolerance response to $\mathrm{Cd}^{2+}$, it was suggested that CNGC19 and CNGC20 play roles in the plant response to toxic heavy metal ions, including $\mathrm{Cd}^{2+}$.

In the present study, we found that several members of the Arabidopsis CNGC family have potential roles in plant tolerance to heavy metals and uptake of $\mathrm{Pb}^{2+}$ and $\mathrm{Cd}^{2+}$. As non-selective cation transporters, CNGCs represent possible entry pathways for heavy metal ions; however, only a few cyclic nucleotide target proteins have been connected to this process [26,37]. The results of the current study indicate that a number of plant CNGC members are potentially involved in heavy metal uptake and tolerance in plants.

\section{Materials and Methods}

\subsection{Plant Material and Growth Condition}

Arabidopsis thaliana L. (Heynh) ecotype Columbia-0 (Col-0) was used in the study, along with a series of T-DNA insertion or single nucleotide substitution mutants for AtCNGCs. Seeds for the mutant 
lines were obtained from the Arabidopsis Biological Resource Center (ABRC) (https://abrc.osu.edu/). The selected mutant lines were: AtCNGC1 (CS874223, atcngc1-1), AtCNGC2 (CS6523), AtCNGC3 (Salk_056832; Salk_066634), AtCNGC4 (CS6524), AtCNGC5 (Salk_149893), AtCNGC6 (Salk_064702; Salk_042207), AtCNGC7 (Salk_060871; CS870639), AtCNGC8 (Salk_008889; Salk_008898), AtCNGC10 (CS859870, atcngc10-1), AtCNGC11 (Salk_026568, atcngc11-1; Salk_085485, atcngc11-2) AtCNGC12 (Salk_092657), AtCNGC13 (Salk_057742, atcngc13-1; Salk_013536, atcngc13-2), AtCNGC14 (CS86592; CS92192), AtCNGC15 (CS93704, atcngc15-1), AtCNGC16 (Salk_065792, atcngc16-1; Salk_053694, atcngc16-2; CS876303, atcngc16-3), AtCNGC17 (Salk_041923; Salk_076540), AtCNGC19 (CS860128, atcngc19-1; CS860131, atcngc19-2), AtCNGC20 (Salk_129133, atcngc20-1; Salk_074919, atcngc20-2) in the Col-0 background. The seeds of all plant lines were surface sterilized with $70 \%(v / v)$ ethanol and $0.05 \%(v / v)$ Triton X-100, then rinsed in Milli-Q water and placed on media containing $1.75 \mathrm{mM} \mathrm{KCl}$, $50 \mu \mathrm{M} \mathrm{H}_{3} \mathrm{BO}_{3}, 10 \mu \mathrm{M} \mathrm{MnCl}_{2}, 2 \mu \mathrm{M} \mathrm{ZnSO}_{4}, 1.5 \mu \mathrm{M} \mathrm{CuSO}_{4}, 0.075 \mu \mathrm{M} \mathrm{NH}_{4} \mathrm{Mo}_{7} \mathrm{O}_{24}, 74 \mu \mathrm{M}$ Fe-EDTA, $0.5 \mathrm{mM}$ phosphoric acid, $2 \mathrm{mM} \mathrm{Ca}\left(\mathrm{NO}_{3}\right)_{2}$, and $0.75 \mathrm{mM} \mathrm{MgSO}_{4}$ at pH 5.8 with $\mathrm{Ca}(\mathrm{OH})_{2}, 1 \%(w / v)$ sucrose, and $1 \%(w / v)$ Agarose L03 (TAKARA, Kusatsu, Japan). Media with $150 \mu \mathrm{M} \mathrm{Pb}\left(\mathrm{NO}_{3}\right)_{2}$, or 55 $\mu \mathrm{M} \mathrm{CdCl}_{2} \cdot 2.5 \mathrm{H}_{2} \mathrm{O}$ were used for applying heavy metal stress. After stratification for 3 days at $4{ }^{\circ} \mathrm{C}$, seeds were germinated and grown on vertically positioned plates in a controlled growth cabinet for 8 days, with a $16 / 8 \mathrm{~h}$ light/dark cycle $\left(80\right.$ to $\left.100 \mu \mathrm{mol} \mathrm{m} \mathrm{m}^{-2} \mathrm{~s}^{-1}\right)$ at $22{ }^{\circ} \mathrm{C}$.

\subsection{Root Growth Assay}

Plants grown for 8 days were photographed and the lengths of the primary roots ( $\geq 80$ seedling plants of each genotype studied for each treatment) were analyzed using ImageJ software (National Institutes of Health, Bethesda, MD, USA). All experiments were performed three times, and a representative set of data are presented. Statistical analysis consisted of a one-way ANOVA with a post-hoc Tukey's comparison using GraphPad Prism (GraphPad Software, San Diego, CA, USA) software.

\subsection{Ion Content}

Three biological replicates consisting of 30-40 pooled seedlings in each replicate were analyzed. Whole eight-day-old plantlets were harvested, rinsed in Milli-Q water, and dried in an oven at $65^{\circ} \mathrm{C}$ for 4 days. Two $\pm 0.1 \mathrm{mg}$ of dried samples were degraded in $1 \mathrm{~mL}$ of $60 \%(v / v) \mathrm{HNO}_{3}$ by heating at $125^{\circ} \mathrm{C}$ for $3 \mathrm{~h}$. The resulting samples were then diluted with Milli-Q water to $10 \mathrm{~mL}$. Elemental levels were measured by inductively coupled plasma mass spectrometry (NexION ${ }^{\circledR} 300$ ICP-MS System, Perkin Elmer, Waltham, MA, USA), and concentrations of the elements were calculated based on dry weight. Statistical differences were evaluated with a T-test using GraphPad Prism software (GraphPad Software, San Diego, CA, USA).

Supplementary Materials: Supplementary materials can be found at http:/ / www.mdpi.com/1422-0067/20/2/ $413 /$ s1.

Author Contributions: J.Y.M. and R.S. participated in the experimental design; J.Y.M., C.B. and M.D.I. carried out plant culture experiments, measurement of root growth and the statistical analysis; J.Y.M. performed elementary extraction and ion content analysis; J.Y.M. wrote the manuscript; and R.S., C.B. and M.D.I. revised and finally approved this article for publication.

Funding: This work was funded by RIKEN Incentive Research Projects (201801062220) and the Ministry of Education, Culture, Sports, Science and Technology [Grants-in-Aid (17K00608)].

Acknowledgments: We are grateful to Eri Adams for valuable discussion, to Takae Miyazaki for assistance for plant experiments, and to New Colombo Plan Scholarship Program (Australian Government) for supporting the Internship program (M.D.I.).

Conflicts of Interest: The authors declare no conflict of interest. 


\section{Abbreviations}

$\begin{array}{ll}\text { CNGC } & \text { Cyclic Nucleotide-Gated Channel } \\ \text { CN } & \text { Cyclic Nucleotide } \\ \text { cAMP } & \text { cyclic Adenosine MonoPhosphate } \\ \text { cGMP } & \text { cyclic Guanosine MonoPhosphate } \\ \text { CNBD } & \text { CN binding domains } \\ \text { CaM } & \mathrm{Ca}^{2+} \text {-activated calmodulin } \\ \text { ROS } & \text { Reactive Oxygen Species }\end{array}$

\section{References}

1. Alloway, B.J. Sources of heavy metals and metalloids in soils. In Heavy Metals in Soil, 3rd ed.; Alloway, B.J., Ed.; Springer: Dordrecht, The Netherlands, 2013; Volume 22, pp. 11-50. ISBN 978-94-007-4469-1.

2. Wu, Q.; Zhou, H.; Tam, N.F.; Tian, Y.; Tan, Y.; Zhou, S.; Li, Q.; Chen, Y.; Leung, J.Y. Contamination, toxicity and speciation of heavy metals in an industrialized urban river: Implications for the dispersal of heavy metals. Mar. Pollut. Bull. 2016, 104, 153-161. [CrossRef] [PubMed]

3. Wu, X.; Cobbina, S.J.; Mao, G.; Xu, H.; Zhang, Z.; Yang, L. A review of toxicity and mechanisms of individual and mixtures of heavy metals in the environment. Environ. Sci. Pollut. Res. Int. 2016, 23, 8244-8259. [CrossRef] [PubMed]

4. Shahid, M.; Pourrut, B.; Dumat, C.; Nadeem, M.; Aslam, M.; Pinelli, E. Heavy-metal-induced reactive oxygen species: Phytotoxicity and physicochemical changes in plants. Rev. Environ. Contam. Toxicol. 2014, 232, 1-44. [CrossRef] [PubMed]

5. Jaishankar, M.; Tseten, T.; Anbalagan, N.; Mathew, B.B.; Beeregowda, K.N. Toxicity, mechanism and health effects of some heavy metals. Interdiscip. Toxicol. 2014, 7, 60-72. [CrossRef] [PubMed]

6. Hansch, R.; Mendel, R.R. Physiological functions of mineral micronutrients ( $\mathrm{Cu}, \mathrm{Zn}, \mathrm{Mn}, \mathrm{Fe}, \mathrm{Ni}, \mathrm{Mo}, \mathrm{B}, \mathrm{Cl})$. Curr. Opin. Plant Biol. 2009, 12, 259-266. [CrossRef] [PubMed]

7. Kanter, U.; Hauser, A.; Michalke, B.; Draxl, S.; Schaffner, A.R. Caesium and strontium accumulation in shoots of Arabidopsis thaliana: Genetic and physiological aspects. J. Exp. Bot. 2010, 61, 3995-4009. [CrossRef] [PubMed]

8. White, P.J. The pathways of calcium movement to the xylem. J. Exp. Bot. 2001, 52, 891-899. [CrossRef]

9. White, P.J.; Davenport, R.J. The voltage-independent cation channel in the plasma membrane of wheat roots is permeable to divalent cations and may be involved in cytosolic $\mathrm{Ca}^{2+}$ homeostasis. Plant Physiol. 2002, 130, 1386-1395. [CrossRef]

10. White, P.J.; Broadley, M.R. Mechanisms of caesium uptake by plants. New Phytol. 2000, 147, $241-256$. [CrossRef]

11. Zhu, Y.G.; Smolders, E. Plant uptake of radiocaesium: A review of mechanisms, regulation and application. J. Exp. Bot. 2000, 51, 1635-1645. [CrossRef]

12. DeFalco, T.A.; Moeder, W.; Yoshioka, K. Opening the gates: Insights into cyclic nucleotide-gated channel-mediated signaling. Trends Plant Sci. 2016, 21, 903-906. [CrossRef] [PubMed]

13. Kaplan, B.; Sherman, T.; Fromm, H. Cyclic nucleotide-gated channels in plants. FEBS Lett. 2007, 581, 2237-2246. [CrossRef]

14. Moeder, W.; Urquhart, W.; Ung, H.; Yoshioka, K. The role of cyclic nucleotide-gated ion channels in plant immunity. Mol. Plant 2011, 4, 442-452. [CrossRef]

15. Talke, I.N.; Blaudez, D.; Maathuis, F.J.; Sanders, D. CNGCs: Prime targets of plant cyclic nucleotide signalling? Trends Plant Sci. 2003, 8, 286-293. [CrossRef]

16. Cukkemane, A.; Seifert, R.; Kaupp, U.B. Cooperative and uncooperative cyclic-nucleotide-gated ion channels. Trends Biochem. Sci. 2011, 36, 55-64. [CrossRef] [PubMed]

17. Matulef, K.; Zagotta, W.N. Cyclic nucleotide-gated ion channels. Annu. Rev. Cell Dev. Biol. 2003, 19, $23-44$. [CrossRef] [PubMed]

18. Spalding, E.P.; Harper, J.F. The ins and outs of cellular $\mathrm{Ca}^{2+}$ transport. Curr. Opin. Plant Biol. 2011, 14, 715-720. [CrossRef] 
19. Arazi, T.; Kaplan, B.; Fromm, H. A high-affinity calmodulin-binding site in a tobacco plasma-membrane channel protein coincides with a characteristic element of cyclic nucleotide-binding domains. Plant Mol. Biol. 2000, 42, 591-601. [CrossRef]

20. Pilot, G.; Pratelli, R.; Gaymard, F.; Meyer, Y.; Sentenac, H. Five-group distribution of the Shaker-like $\mathrm{K}^{+}$ channel family in higher plants. J. Mol. Evol. 2003, 56, 418-434. [CrossRef]

21. Leng, Q.; Mercier, R.W.; Hua, B.G.; Fromm, H.; Berkowitz, G.A. Electrophysiological analysis of cloned cyclic nucleotide-gated ion channels. Plant Physiol. 2002, 128, 400-410. [CrossRef]

22. Leng, Q.; Mercier, R.W.; Yao, W.; Berkowitz, G.A. Cloning and first functional characterization of a plant cyclic nucleotide-gated cation channel. Plant Physiol. 1999, 121, 753-761. [CrossRef]

23. Schuurink, R.C.; Shartzer, S.F.; Fath, A.; Jones, R.L. Characterization of a calmodulin-binding transporter from the plasma membrane of barley aleurone. Proc. Natl. Acad. Sci. USA 1998, 95, 1944-1949. [CrossRef] [PubMed]

24. Ward, J.M.; Maser, P.; Schroeder, J.I. Plant ion channels: Gene families, physiology, and functional genomics analyses. Annu Rev. Physiol. 2009, 71, 59-82. [CrossRef] [PubMed]

25. Arazi, T.; Kaplan, B.; Sunkar, R.; Fromm, H. Cyclic-nucleotide- and $\mathrm{Ca}^{2+} /$ calmodulin-regulated channels in plants: Targets for manipulating heavy-metal tolerance, and possible physiological roles. Biochem. Soc. Trans. 2000, 28, 471-475. [CrossRef] [PubMed]

26. Arazi, T.; Sunkar, R.; Kaplan, B.; Fromm, H. A tobacco plasma membrane calmodulin-binding transporter confers $\mathrm{Ni}^{2+}$ tolerance and $\mathrm{Pb}^{2+}$ hypersensitivity in transgenic plants. Plant J. 1999, 20, 171-182. [CrossRef] [PubMed]

27. Kohler, C.; Merkle, T.; Neuhaus, G. Characterisation of a novel gene family of putative cyclic nucleotide- and calmodulin-regulated ion channels in Arabidopsis thaliana. Plant J. 1999, 18, 97-104. [CrossRef] [PubMed]

28. Kohler, C.; Neuhaus, G. Characterisation of calmodulin binding to cyclic nucleotide-gated ion channels from Arabidopsis thaliana. FEBS Lett. 2000, 471, 133-136. [CrossRef]

29. Maser, P.; Thomine, S.; Schroeder, J.I.; Ward, J.M.; Hirschi, K.; Sze, H.; Talke, I.N.; Amtmann, A.; Maathuis, F.J.; Sanders, D.; et al. Phylogenetic relationships within cation transporter families of Arabidopsis. Plant Physiol. 2001, 126, 1646-1667. [CrossRef]

30. Urquhart, W.; Chin, K.; Ung, H.; Moeder, W.; Yoshioka, K. The cyclic nucleotide-gated channels AtCNGC11 and 12 are involved in multiple $\mathrm{Ca}^{2+}$-dependent physiological responses and act in a synergistic manner. J. Exp. Bot. 2011, 62, 3671-3682. [CrossRef]

31. Balague, C.; Lin, B.; Alcon, C.; Flottes, G.; Malmstrom, S.; Kohler, C.; Neuhaus, G.; Pelletier, G.; Gaymard, F.; Roby, D. HLM1, an essential signaling component in the hypersensitive response, is a member of the cyclic nucleotide-gated channel ion channel family. Plant Cell 2003, 15, 365-379. [CrossRef]

32. Chan, C.W.; Schorrak, L.M.; Smith, R.K., Jr.; Bent, A.F.; Sussman, M.R. A cyclic nucleotide-gated ion channel, CNGC2, is crucial for plant development and adaptation to calcium stress. Plant Physiol. 2003, 132, 728-731. [CrossRef] [PubMed]

33. Ma, W.; Ali, R.; Berkowitz, G.A. Characterization of plant phenotypes associated with loss-of-function of AtCNGC1, a plant cyclic nucleotide gated cation channel. Plant Physiol. Biochem. 2006, 44, 494-505. [CrossRef] [PubMed]

34. Gobert, A.; Park, G.; Amtmann, A.; Sanders, D.; Maathuis, F.J. Arabidopsis thaliana cyclic nucleotide gated channel 3 forms a non-selective ion transporter involved in germination and cation transport. J. Exp. Bot. 2006, 57, 791-800. [CrossRef] [PubMed]

35. Li, X.; Borsics, T.; Harrington, H.M.; Christopher, D.A. Arabidopsis AtCNGC10 rescues potassium channel mutants of E. coli, yeast and Arabidopsis and is regulated by calcium/calmodulin and cyclic GMP in E. coli. Funct. Plant Biol. 2005, 32, 643-653. [CrossRef]

36. Guo, K.M.; Babourina, O.; Christopher, D.A.; Borsic, T.; Rengel, Z. The cyclic nucleotide-gated channel AtCNGC10 transports $\mathrm{Ca}^{2+}$ and $\mathrm{Mg}^{2+}$ in Arabidopsis. Physiol. Plant. 2010, 139, 303-312. [CrossRef] [PubMed]

37. Sunkar, R.; Kaplan, B.; Bouche, N.; Arazi, T.; Dolev, D.; Talke, I.N.; Maathuis, F.J.; Sanders, D.; Bouchez, D.; Fromm, H. Expression of a truncated tobacco NtCBP4 channel in transgenic plants and disruption of the homologous Arabidopsis CNGC1 gene confer $\mathrm{Pb}^{2+}$ tolerance. Plant J. 2000, 24, 533-542. [CrossRef]

38. Ohki, S.; Ikura, M.; Zhang, M. Identification of $\mathrm{Mg}^{2+}$-binding sites and the role of $\mathrm{Mg}^{2+}$ on target recognition by calmodulin. Biochemistry 1997, 36, 4309-4316. [CrossRef] 
39. Ouyang, H.; Vogel, H.J. Metal ion binding to calmodulin: NMR and fluorescence studies. Biometals 1998, 11, 213-222. [CrossRef]

40. Simons, T.J.; Pocock, G. Lead enters bovine adrenal medullary cells through calcium channels. J. Neurochem. 1987, 48, 383-389. [CrossRef]

41. Tomsig, J.L.; Suszkiw, J.B. Permeation of $\mathrm{Pb}^{2+}$ through calcium channels: Fura-2 measurements of voltageand dihydropyridine-sensitive $\mathrm{Pb}^{2+}$ entry in isolated bovine chromaffin cells. Biochim. Biophys. Acta 1991, 1069, 197-200. [CrossRef]

42. Genger, R.K.; Jurkowski, G.I.; McDowell, J.M.; Lu, H.; Jung, H.W.; Greenberg, J.T.; Bent, A.F. Signaling pathways that regulate the enhanced disease resistance of Arabidopsis "defense, no death" mutants. Mol. Plant-Microbe Interact. 2008, 21, 1285-1296. [CrossRef] [PubMed]

43. Yuen, C.Y.; Christopher, D.A. The group IV-A cyclic nucleotide-gated channels, CNGC19 and CNGC20, localize to the vacuole membrane in Arabidopsis thaliana. AoB Plants 2013, 5, plt012. [CrossRef]

44. Sharma, S.S.; Dietz, K.J.; Mimura, T. Vacuolar compartmentalization as indispensable component of heavy metal detoxification in plants. Plant Cell Environ. 2016, 39, 1112-1126. [CrossRef] [PubMed]

45. Kugler, A.; Kohler, B.; Palme, K.; Wolff, P.; Dietrich, P. Salt-dependent regulation of a CNG channel subfamily in Arabidopsis. BMC Plant Biol. 2009, 9, 140. [CrossRef] [PubMed]

46. Yuen, C.Y.; Christopher, D.A. The role of cyclic nucleotide-gated channels in cation nutrition and abiotic stress. In Ion Channels and Plant Stress Responses; Demidchik, V., Maathuis, F., Eds.; Springer: Berlin, Germany, 2010; Volume 5, pp. 137-157. ISBN 978-3-642-10493-0.

(C) 2019 by the authors. Licensee MDPI, Basel, Switzerland. This article is an open access article distributed under the terms and conditions of the Creative Commons Attribution (CC BY) license (http://creativecommons.org/licenses/by/4.0/). 